\title{
Distress severity in perceptual anomalies moderates the relationship between prefrontal brain structure and psychosis proneness in nonclinical individuals
}

\author{
Ulrika Evermann ${ }^{1,2} \mathbb{D} \cdot$ Simon Schmitt ${ }^{1,2} \mathbb{D} \cdot$ Tina Meller $^{1,2} \mathbb{D} \cdot$ Julia-Katharina Pfarr $^{1,2} \mathbb{D} \cdot$ Sarah Grezellschak $^{1,2,3}$. \\ Igor Nenadić ${ }^{1,2,3}$
}

Received: 14 August 2020 / Accepted: 18 December 2020 / Published online: 2 February 2021

(c) The Author(s) 2021

\begin{abstract}
In the general population, psychosis risk phenotypes occur independently of attenuated prodromal syndromes. Neurobiological correlates of vulnerability could help to understand their meaningfulness. Interactions between the occurrence of psychotic-like experiences (PLE) and other psychological factors e.g., distress related to PLE, may distinguish psychosis-prone individuals from those without risk of future psychotic disorder. We aimed to investigate whether (a) correlates of total PLE and distress, and (b) symptom dimension-specific moderation effects exist at the brain structural level in non-help-seeking adults reporting PLE below and above the screening criterion for clinical high-risk (CHR). We obtained T1-weighted wholebrain MRI scans from 104 healthy adults from the community without psychosis CHR states for voxel-based morphometry (VBM). Brain structural associations with PLE and PLE distress were analysed with multiple linear regression models. Moderation of PLE by distress severity of two types of positive symptoms from the Prodromal Questionnaire (PQ-16) screening inventory was explored in regions-of-interest after VBM. Total PQ-16 score was positively associated with grey matter volume (GMV) in prefrontal regions, occipital fusiform and lingual gyri $(p<0.05$, FDR peak-level corrected). Overall distress severity and GMV were not associated. Examination of distress severity on the positive symptom dimensions as moderators showed reduced strength of the association between PLE and rSFG volume with increased distress severity for perceptual PLE. In this study, brain structural variation was related to PLE level, but not distress severity, suggesting specificity. In healthy individuals, positive relationships between PLE and prefrontal volumes may indicate protective features, which supports the insufficiency of PLE for the prediction of CHR. Additional indicators of vulnerability, such as distress associated with perceptual PLE, change the positive brain structure relationship. Brain structural findings may strengthen clinical objectives through disentanglement of innocuous and risk-related PLE.
\end{abstract}

Keywords Psychological distress $\cdot$ Psychotic-like experiences $\cdot$ Prodromal questionnaire $\cdot$ Psychosis risk

\section{Introduction}

Ulrika Evermann

ulrika.evermann@staff.uni-marburg.de

Igor Nenadić

nenadic@staff.uni-marburg.de

1 Cognitive Neuropsychiatry Lab, Department of Psychiatry and Psychotherapy, Philipps-Universität Marburg, Rudolf-Bultmann-Str. 8, 35039 Marburg, Germany

2 Center for Mind, Brain and Behavior (CMBB), Hans-Meerwein-Str. 6, 35032 Marburg, Germany

3 Marburg University Hospital-UKGM, Rudolf-Bultmann-Str. 8, 35039 Marburg, Germany
Prevention of psychosis spectrum disorders relies on early risk detection [1]. Prediction of transition to psychosis is particularly enhanced when clinically validated assessments are employed in targeted samples found in specialised mental health services [2]. On the other hand, the use of instruments to assess clinical high risk (CHR) states in general non-help-seeking populations produces weak predictive estimates of the true risk for imminent psychosis [3]. This shortcoming has been encountered by psychometric developments building on two-staged assessments of psychosis CHR states by screening and semi-structured clinical interviews, which enables improved clinical efficiency and 
accuracy [4]. Originally validated in a general mental health help-seeking population, the abbreviated 16-item version of the Prodromal Questionnaire [5] (PQ-16) sufficiently screens for psychosis ultra-high risk (UHR) states [6]. Together the Prodromal Questionnaires (92, 21, and 16-item versions) [5-7] are among the most widely used CHR screening tools [8]. Previous studies have employed the PQ-16 among helpseeking adults [9] and adolescents [10], as well as nonclinical populations [11, 12] for a review see Ref. [13].

The prevalence of subclinical psychotic experiences exceeds that of psychosis in the general population [14], but self-reported psychotic-like experiences (PLE) themselves constitute an inadequate criterion for attenuated psychotic syndromes [15]. Besides clinical prodromal symptoms, screening inventories such as PQ-16, therefore, capture PLE in a broader perspective. Among CHR individuals, motivation to seek help for distressful prodromal symptoms is increased by the burden of affective symptoms leading to greater functional decline [16]; these factors are also captured by semi-structured interviews for attenuated psychotic syndromes [17]. In the general population, evidence exists that persistence of PLE [18], distress [19, 20], and emotional context [21], depression, and reduced functioning [22] associated with positive PLE indicate elevated clinical relevance. The importance of distress for the differentiation between PLE with reduced clinical significance as, for instance, in developmental cohorts [23] and attenuated psychosis risk was also reflected in the uptake of an additional distress severity subscale to the prodromal screening inventory [7].

Multiple neuroimaging studies compared brain morphology in ultra-high risk (UHR) for psychosis to healthy controls or first-episode psychosis patients [24-27]. In contrast to case-control brain imaging studies, which have focused on UHR and first-episode psychosis [28], the nonclinical spectrum (i.e., the occurrence of sparse PLE in healthy subjects) has received less attention despite recent findings of dimensional relations on the phenotype level $[29,30]$. A continuous relationship between infrequent psychotic-like or subclinical symptoms towards a clinical spectrum [31-33] permits a hypothesised relation to neural markers that have been associated with CHR or disease status. This may add to the current understanding of the brain-behaviour relationships in the psychosis spectrum and the development of biomarkers in the early intervention field. Previous studies report associations between subclinical psychotic experiences and brain volume, as well as functional and cortical surface variation [34, 35], some of which converge with alterations typically found in the manifest psychosis spectrum and affective disorders [36]. Across the literature, PLE are associated with structural change in diverse cortical regions, e.g., orbitofrontal and medial temporal lobes [37] and the parietal regions [38]. However, a strong effect for PLE associated with any particular cortical regions derived by meta-analysis is presently lacking. A recent study from our group showed consistent relationships with volume reductions in prefrontal and anterior cingulate regions across multidimensional schizotypy [39], representing a trait-level schizophrenia endophenotype $[40,41]$. Furthermore, the relationship between positive schizotypy and PLE [42-44] is considered to reflect biological psychosis-prone components within schizophrenia endophenotypes [45, 46]. Thus, extending the search for neurobiological correlates relating to PLE may shed further light on the dopaminergic fronto-striatal pathway $[47,48]$ in nonclinical psychosis phenotypes [39, 49].

Building on previous studies [34, 36, 39], we replicate dimensional approaches using whole-brain voxel-wise analysis. Complementary regional analyses are based on primary outcomes to achieve robust targets relevant to the study cohort. The first aim of this investigation was to examine associations between PLE, PLE distress severity, and brain structure. We predict brain structural reductions in association with subclinical PLE and distress severity. Furthermore, we explored the influence of the interaction of PLE and PLE-related distress severity on regional brain volume.

\section{Methods}

\section{Sample}

A total of 104 participants ( 71 females, 33 males; mean age $=24.96, \mathrm{SD}=4.76, \min =18, \max =40$ ), all fluent speakers of the German language, were recruited from the local community using advertisements and the university email circulation service. The study protocol adhered to the Declaration of Helsinki [50] and was approved by the local ethics committee of the School of Medicine, Philipps-University of Marburg. Based on an initial telephone screening protocol, we obtained information on exclusion criteria: medical history (neurological or untreated chronic medical condition), past and current substance use, and any history of psychiatric or neurological disorders and treatments including psychotropic medication. Participants aged $18-40$ years were then screened using the German version of the Structured Clinical Interview for DSM-IV (SCID-I) [51]. Participants provided written informed consent once invited to complete brain scans and online questionnaires [52], and received financial compensation after participation. Mean laterality quotient of handedness [53] within this cohort was 71.91 ( $\mathrm{SD}=62.07$ ). An estimated intelligence quotient (IQ) [54] below 80 was exclusionary. The mean IQ estimate was $117.46(\mathrm{SD}=14.66)$. 


\section{Prodromal Questionnaire (PQ-16)}

We assessed PLE using the 16-item Prodromal Questionnaire (PQ-16) [6], a self-report measure to assess presence of PLE developed from prior versions [5, 7]. The validation of the 16-item version showed that a cutoff of $\geq 6$ endorsed PLE identifies UHR states with $87 \%$ sensitivity and $87 \%$ specificity [6]. Complementary to the total sum of item endorsements on the 2-point scale ('true'/'false'), a measure of distress severity for each endorsed item is obtained on a 4-point scale from 0 ('none') to 3 ('severe'). In addition to the total symptom score, the distress severity scale cutoff score $\geq 9$ was recommended in a study of non-help-seeking subjects [55]. Based on previous psychometric studies [5, 6, 23, 56] and guided by item comparison to the German version of the Structured Interview for Prodromal Syndromes Version 5.0 (SIPS) [17], we assigned items to two positive symptom subscales reflecting 'Perceptual abnormalities/Hallucinations' (Perceptual: items 3, 4, 5, 6, 8, 9, 12, 13, 15), 'Unusual thought content/Delusional ideas' (Delusional: items 2, 10, 11, 14, 16), and Negative symptoms (items 1 and 7)
(Table 1). Table 2 displays Cronbach's alpha as measures of internal consistency for these scales, and frequency of

Table 2 Reliability measures for subscales derived from the Prodromal Questionnaire (PQ-16)

\begin{tabular}{llllll}
\hline PQ-16 scale & Min & Max & Skew & Kurtosis & $\alpha^{\mathrm{a}}$ \\
\hline Total PLE & 0 & 9 & 1.84 & 3.65 & 0.69 \\
Total PLE distress & 0 & 10 & 1.77 & 2.79 & 0.58 \\
Perceptual scale & & & & & \\
$\quad$ Total & 0 & 5 & 2.79 & 8.48 & 0.62 \\
$\quad$ Distress & 0 & 6 & 2.61 & 6.81 & 0.47 \\
$\quad$ Delusional scale & & & & & \\
$\quad$ Total & 0 & 4 & 1.51 & 1.84 & 0.46 \\
$\quad$ Distress & 0 & 5 & 1.79 & 2.88 & 0.28 \\
$\quad$ Negative scale & & & & & \\
$\quad$ Total & 0 & 2 & 1.79 & 2.20 & 0.13 \\
$\quad$ Distress & 0 & 3 & 3.01 & 10.00 & 0.18 \\
\hline
\end{tabular}

${ }^{\mathrm{a}} \alpha=$ Cronbach's alpha

Table 1 Descriptive statistics of PLE in 104 healthy adults assessed by Prodromal Questionnaire (PQ-16)

\begin{tabular}{|c|c|c|c|c|c|c|}
\hline \multirow[t]{2}{*}{ Prodromal questionnaire (PQ-16) } & \multicolumn{2}{|c|}{ Total scale } & \multicolumn{4}{|c|}{ Distress scale } \\
\hline & Mean & $\mathrm{SD}^{\mathrm{a}}$ & Mean & $\mathrm{SD}^{\mathrm{a}}$ & $r^{\mathrm{b}}$ & $p_{F D R}{ }^{\mathrm{c}}$ \\
\hline PLE score & 1.30 & 1.78 & 1.44 & 2.15 & 0.92 & $<0.001$ \\
\hline Perceptual abnormalities/Hallucinations & 0.50 & 1.01 & 0.56 & 1.21 & 0.68 & $<0.001$ \\
\hline I sometimes smell or taste things that other people can't smell or taste. & 0.09 & 0.28 & 0.09 & 0.34 & 0.37 & $<0.001$ \\
\hline I often hear unusual sounds like banging, clicking, hissing, clapping or ringing in my ears. & 0.07 & 0.25 & 0.08 & 0.39 & 0.33 & 0.001 \\
\hline I have been confused at times whether something I experienced was real or imaginary. & 0.04 & 0.19 & 0.06 & 0.31 & 0.31 & 0.001 \\
\hline $\begin{array}{l}\text { When I look at a person, or look at myself in a mirror, I have seen the face change right before my } \\
\text { eyes. }\end{array}$ & 0.02 & 0.14 & 0.02 & 0.14 & 0.22 & 0.026 \\
\hline I have seen things that other people apparently can't see. & 0.02 & 0.14 & 0.02 & 0.14 & 0.24 & 0.019 \\
\hline My thoughts are sometimes so strong that I can almost hear them. & 0.12 & 0.32 & 0.14 & 0.51 & 0.37 & $<0.001$ \\
\hline Sometimes I feel suddenly distracted by distant sounds that I am not normally aware of. & 0.10 & 0.30 & 0.10 & 0.33 & 0.39 & $<0.001$ \\
\hline I have heard things other people can't hear like voices of people whispering or talking. & 0.01 & 0.10 & 0.02 & 0.20 & 0.15 & 0.126 \\
\hline I have had the sense that some person or force is around me, even though I could not see anyone. & 0.05 & 0.21 & 0.04 & 0.19 & 0.36 & $<0.001$ \\
\hline Unusual thought content/Delusional ideas & 0.59 & 0.89 & 0.65 & 1.10 & 0.75 & $<0.001$ \\
\hline I often seem to live through events exactly as they happened before (déjà vu). & 0.23 & 0.42 & 0.26 & 0.61 & 0.47 & $<0.001$ \\
\hline $\begin{array}{l}\text { I sometimes see special meanings in advertisements, shop windows, or in the way things are } \\
\text { arranged around me. }\end{array}$ & 0.12 & 0.32 & 0.12 & 0.43 & 0.48 & $<0.001$ \\
\hline Sometimes I have felt that I'm not in control of my own ideas or thoughts. & 0.09 & 0.28 & 0.10 & 0.38 & 0.37 & $<0.001$ \\
\hline I often feel that others have it in for me. & 0.13 & 0.34 & 0.18 & 0.50 & 0.45 & $<0.001$ \\
\hline $\begin{array}{l}\text { I feel that parts of my body have changed in some way, or that parts of my body are working dif- } \\
\text { ferently than before. }\end{array}$ & 0.02 & 0.14 & 0.00 & 0.00 & 0.21 & 0.033 \\
\hline Negative symptoms & 0.21 & 0.43 & 0.23 & 0.58 & 0.47 & $<0.001$ \\
\hline I feel uninterested in the things I used to enjoy. & 0.19 & 0.40 & 0.21 & 0.53 & 0.43 & $<0.001$ \\
\hline I get extremely anxious when meeting people for the first time. & 0.02 & 0.14 & 0.02 & 0.14 & 0.25 & 0.015 \\
\hline
\end{tabular}

${ }^{\mathrm{a}} \mathrm{SD}=$ standard deviation

${ }^{\mathrm{b}} r=$ Spearman correlation coefficient

${ }^{\mathrm{c}} p_{\mathrm{FDR}}=p$-value after false discovery rate (FDR) adjustment 
single item endorsements within this community sample is shown in Fig. 1.

\section{MRI acquisition and voxel-based morphometry (VBM)}

We obtained high-resolution T1-weighted MRI using a 3.0-T Siemens Tim Trio scanner (Siemens, Erlangen, Germany) with standard 12-channel quadrature head coil and a 3D magnetisation-prepared rapid-acquisition gradient echo (MP-RAGE) sequence (4:26 $\mathrm{min} ; \mathrm{TE}=2.26 \mathrm{~ms}$, $\mathrm{TI}=900 \mathrm{~ms}, \mathrm{TR}=1900 \mathrm{~ms}, 1 \mathrm{~mm}^{3}$ isotropic voxel resolution). We then used the Computational Anatomy Toolbox for SPM (CAT12 v12.6, r1450, Christian Gaser, Structural Brain Mapping Group, Jena University Hospital, Germany) in SPM12 (v7219, Statistical Parametric Mapping, Wellcome Trust Centre for Neuroimaging, London, UK) for correction of homogeneity bias and segmentation of T1-weighted images into grey (GM) and white matter and cerebrospinal fluid. All images passed both visual inspection and CAT12 quality assessment protocols. Internal GM threshold was set to 0.1 and scans were smoothed with a full width at half maximum Gaussian kernel of $8 \mathrm{~mm}$.

\section{Statistical analyses: general linear models}

Multiple linear regression models were conducted in SPM12 (v7487) running in Matlab (R2017a, The
Mathworks Inc., USA) to test associations between grey matter volume (GMV) and total PLE score and distress severity score, respectively. Age, sex, and total intracranial volume (TIV) were entered as control variables to these models. In these voxel-wise volumetric analyses, the statistical threshold was set to $p<0.05$ applying falsediscovery-rate (FDR) peak-level correction. Anatomical labelling of maximum voxel coordinates was based on the DARTEL neuromorphometrics atlas.

\section{Moderation analyses}

Using the regions-of-interest tool within CAT12.5 (r1363), we extracted estimated mean GMV for each participant based on the neuromorphometrics atlas. These volumes of interest (VOI) were dependent variables in moderation analyses conducted in PROCESS 3.3 [57] for SPSS (Version 25.0, IBM Corp., Armonk, NY). Interactions of Total PLE $\times$ distress for Perceptual and Delusional distress severity were examined as estimators of VOI. Due to the low item and score range, we refrained from including Negative symptoms in moderation analyses. We corrected coefficient $p$ values for multiple comparisons for the number of dependent variables (VOI) for each PLE subscale using FDR adjusted $p$ values. FDR corrections for multiple comparisons [58] were carried out in $\mathrm{R}$ [59].

\section{Delusional $\square$ Negative $\square$ Perceptual}

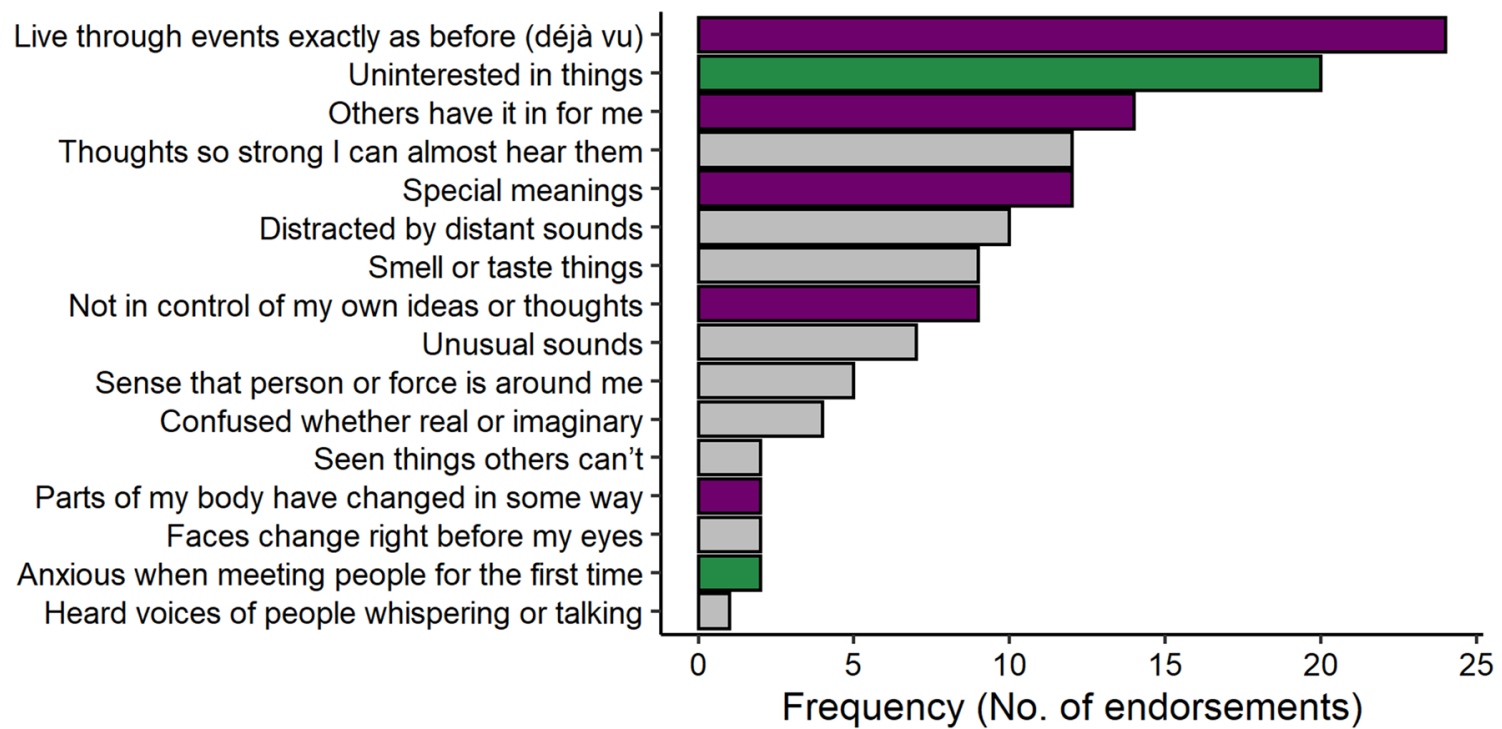

Fig. 1 Distribution of psychotic-like experiences (PLE) captured by the German version of the Prodromal Questionnaire 16 (PQ-16) in 104 nonclinical subjects. Most PLE were assigned to three categories reflecting positive (Delusional, Perceptual) and Negative PLE based on a comparison to the Structured Interview for Prodromal Syndromes (SIPS). Note: Item descriptions are abbreviated for display purposes. This figure was created using ggplot2 [94] 


\section{Results}

\section{PLE screening outcomes}

On average, at least one PLE $(M=1.30, \mathrm{SD}=1.78$, scale score range $=0-9)$ and a mean distress dimension score of $1.44(\mathrm{SD}=2.15$, scale score range $=0-10)$ was reported in the present sample. Table 1 provides descriptive statistics for each PQ-16 item, the three PLE subscales and correlations with the overall distress score with two-sided significance levels. Four participants met the clinical screening threshold (PQ-16 total score $\geq 6$ and/or PQ-16 distress score $\geq 9$ ) and were invited to a follow-up assessment for CHR status using Schizophrenia Proneness Instrument, Adult version (SPI-A) [60]. Three participants completed the clinical interview; none met basic symptom criteria.

\section{VBM outcomes for PLE}

Total PLE score showed a significant positive association with volume in the right prefrontal region (cluster size $k=246$ ) with two significant peaks at the right superior (rSFG, maximum voxel coordinates $X / Y / Z=18 /-3 / 56$, $t=5.42, p=0.009)$ and middle frontal gyrus (rMFG) (maximum voxel coordinates $X / Y / Z=30 / 2 / 52, t=5.97$, $p=0.005)$. PQ-16 associations were significant at the FDR-corrected statistical threshold in the occipital fusiform and lingual gyri $(k=45, X / Y / Z=22 /-76 /-14$, $t=4.73, p=0.019)$, in another small cluster in the rMFG $(k=6, X / Y / Z=-34 / 22 / 45, t=4.40, p=0.031)$ and left precentral gyrus $(k=1, X / Y / Z=-39 / 0 / 46, t=4.18$, $p=0.048$ ) (all FDR-corrected $p$-values) (Fig. 2). Distress severity showed no positive or negative relationship with GMV after FDR correction for statistical significance.

\section{Moderating effects of PLE distress severity}

The prefrontal VBM cluster showed two local maxima in the dorsolateral prefrontal cortex (DLPFC), indicating the middle (rMFG) and superior frontal gyri (rSFG). For Delusional, no effect was observed in either rMFG or rSFG model. A moderating effect of Perceptual in the rMFG was only significant at trend-level [unstandardized coefficient $=-0.15, \mathrm{SE}=0.08, t(97)=-1.90$, $\left.p_{\text {FDR }}=0.060\right]$, while the overall significant model for the $\operatorname{rSFG}\left[F(6,97)=22.06, p<0.001, R^{2}=0.52\right]$ showed a significant moderation of Perceptual distress scores $\geq 2.75$ [unstandardised coefficient $=-0.09, \mathrm{SE}=0.04$, $\left.t(97)=-2.32, p_{\mathrm{FDR}}=0.044\right]$, with increased Perceptual distress resulting in decreased GM value (Table 3). Due to an overrepresentation of females, the additional nonsignificant moderating effect of sex on this pathway (i.e. PLE $\times$ Perceptual PLE distress $\times$ Sex interaction) was inspected with the PROCESS macro.

\section{Discussion}

The present study aimed to elucidate the relationship between brain structure and PLE in nonclinical subjects devoid of attenuated risk for psychosis. The results revealed a positive association between PLE and volume in right dorsolateral prefrontal, fusiform and occipital brain regions, which was not present for the distress severity scale. However, exploratory analysis of the whole right superior and middle frontal gyral volumes showed a modulating effect of distress severity.

The main finding of this study is that PLE applicable for psychosis risk screening are associated with neurobiological changes independent of UHR case-control status, conversion [61], and UHR phenotype heterogeneity (e.g., genetic risk deterioration syndrome, attenuated psychotic syndrome, brief limited intermittent psychotic symptoms) [62]. Correlates for subclinical PLE were detected in the right hemisphere. This differs from clinical findings in schizophrenia, showing either left lateral or bilateral GM reductions in the medial and superior temporal lobes $[63,64]$ and a linkage with severity of auditory hallucinations [65]. However, GM alterations in the right dorsolateral prefrontal cortex are also represented in studies of schizophrenia and diverse prodromal stages [63, 66-69]. Regional GM differences between healthy, genetic-high risk, and first-episode schizophrenia individuals also highlight genetic components [70]. Our significant regional findings align with some of those found in the genetic-high risk group in Chang et al.[70], such as larger volumes in rMFG and fusiform gyrus compared to healthy controls. Interestingly, a large genome-wide association study recently demonstrated shared genetic liability between PLE and multiple psychiatric conditions [71].

Magnitude of GMV loss shows some variability over disease progression [72], and progressive structural differences were also seen in reduced white matter growth in UHR adolescents [73]. Accelerated prefrontal GMV loss may indicate differential pathological processes at different neurodevelopmental stages in schizophrenia [74]. This would be in line with potentially non-linear patterns of brain structural changes dependent on transition and illness phase [75]. However, another comparison of CHR youths to controls could not confirm structural and cortical thickness differences regardless of later transition to psychosis [76]. In that study, the critical role of sample uniqueness, especially the absence of illicit drug use, including cannabis, are discussed. 



Right middle frontal gyrus [Peak coordinate $=30 / 2 / 52(t=5.97)]$ Right superior frontal gyrus [Peak coordinate $=18 /-3 / 56(t=5.42)]$

Perceptual PLE distress severity $\begin{array}{lllllll}0 & 1 & 2 & 3 & 4 & 5 & 6\end{array}$


Fig. 2 Upper panel shows statistical significance (thresholded at FDR-corrected $p<0.05$ ) and effect size (thresholded at uncorrected peak-level $p<0.001$ ) maps for structural correlates of total psychoticlike experiences (PLE), assessed by the Prodromal Questionnaire (PQ-16). Mean volumes of interest (VOI) were extracted from two prefrontal regions: right superior (rSFG) and middle frontal gyri (rMFG), which enclose the largest cluster of size $k=246$. Lower

An extension of our design would be an exploration of the effect of illicit drug use on the observed PLE-brain structural relationship. panel shows the effect of distress and PLE interaction on predicted rSFG volume. At higher Perceptual PLE distress severity (scale score $\geq 2.75$ ), overall PLE are associated with predicted rSFG volume reductions. This figure was created using MRIcroGL (https://www. mccauslandcenter.sc.edu/mricrogl/), ggplot2 [94], 3D Slicer (https:// www.slicer.org) [95] and GNU Image Manipulation Program (GIMP, https://www.gimp.org/)

Contrary to predictions, we found a positive direction for the association between PLE and GMV. In the earlier analysis [39], positive schizotypal traits were associated with GMV reductions in superior and middle frontal gyri. 


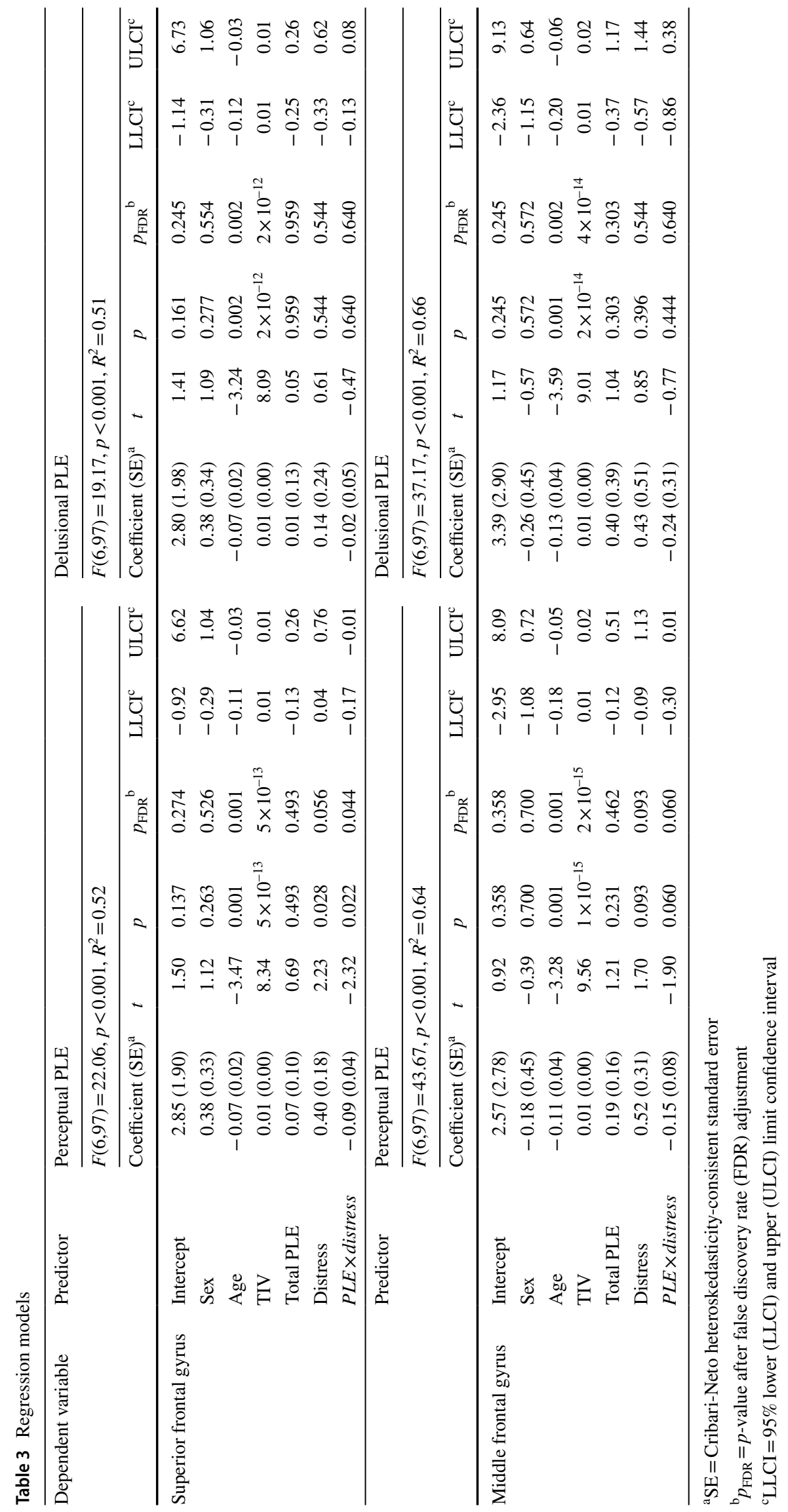


Tract-based white matter and GMV analyses implicated alterations in fronto-striatal network regions in schizotypy. However, it remains speculative whether all schizotypy dimensions equally reflect neural deficits or vulnerability. The proximity between PLE and positive schizotypy is further supported by their anatomical overlap, however, PLE correlated with larger volumes in a prefrontal cluster. Together the findings from these two studies do not support a linear continuum ranging from the subclinical phenotypes to CHR and schizophrenia spectrum disorders $[31,77]$.

The right superior and middle frontal gyri, which are cortical correlates in CHR and transition status [26, 78], could imply modulation by intraindividual psychological factors that may convey vulnerability or resilience in nonclinical individuals, too. Larger DLPFC volumes may be explained by compensatory mechanisms, e.g., in response to upstream striatal alterations [77]. Compensatory processes were also proposed for larger precuneus and posterior cingulate cortex volumes in association with nonclinical psychosis proneness $[34,79,80]$, despite volume reductions in the clinical spectrum being common $[68,81,82]$. In that case, larger regional prefrontal volumes at higher PLE levels, but reductions related to the interaction of overall PLE and distress severity of perceptual anomalies, may indicate attenuated protective features. This buffering explanation was earlier proposed by Meller et al. [49], showing that the association between positive schizotypy and larger striatal volume is decreased by general intelligence (a functional substrate of the frontal regions). Preservation of prefrontal functions and GMV [83] may be pivotal determinants of clinical deterioration and prevention. A comparison of brain developmental trajectories in resilient and non-resilient UHR youths found larger frontal volumes over time in the higher functioning group [27]. Resilience [84] may contribute to prefrontal cortical variation in nonclinical subjects as well. This would be in keeping with the notion that PLE are manifestations of the positive schizotypy dimension [43], which correlates with psychosis-relevant genotypes involved in dopamine regulation [45]. A specific effect for the perceptual PLE component also fits in with the striatal dopamine hypothesis underlying psychosis in schizophrenia and general psychosis proneness [47].

Additionally, the right occipital fusiform and lingual regions were positively associated with PLE. This finding in the occipitotemporal region indicates unique PLE correlates that were not present in multidimensional schizotypy. Involvement of the fusiform gyrus in perception and face recognition $[85,86]$, together with occipitotemporal GMV reductions in schizophrenia and psychosis [87-89], underpins deficits related to facial processing in the clinical spectrum [90]. One PQ-16 item ('When I look at a person, or look at myself in a mirror, I have seen the face change right before my eyes') may have been especially relevant to the diametrically opposed outcome in nonclinical individuals. Another study reported associations between positive PLE distress and precuneus volume, which were not present in trait psychosis proneness [38]. Our findings for a positive association for PLE load located in the dorsolateral cortical regions as opposed to parietal brain regions may be explained by differences between purely quantitative PLE levels, and measures relating to the qualitative burden of PLE. Failure to replicate precuneus correlates for PLE distress in the present study may be attributed to differing psychometric PLE measures related to different aspects of psychosis proneness. Nonetheless, they complement each other in that they underline the impact of perceiving positive symptoms as worrisome in brain regions implicated across the psychosis spectrum.

Some limitations of this study require evaluation. Although the present cohort consists of young adults, we must acknowledge that cross-sectional designs do not permit prediction of subsequent psychopathological development. Another inherent problem of studies with nonclinical designs is a non-normal PLE distribution [91]. Also, the size of the study cohort was limited, which might have hampered the detection of smaller effects. Adoption of instrument (long vs. short PQ versions), setting, and UHR enrichment are sources of detection threshold variability [13]. Self-reported PLE are poor measures of clinician-rated psychosis risk [15], and current recommendations state clinical CHR assessment should only be extended to those distressed by symptoms [1]. Note also that symptom dimensions were based on the assessment of item contents but require validation using factor analysis. This is especially recommended for positive items where the latent delusional or perceptual character is ambiguous. Brandizzi and colleagues' [23] analysis of PQ-92 positive items yielded factors reflecting 'perceptual abnormalities', 'bizarre experiences', as well as 'conceptual disorganisation and suspiciousness' and 'magical ideation' also found in schizotypy. Additionally, Kotzalidis et al. [56] identified a four-factor solution, including a heterogeneous 'functional' dimension. While this provides options for replication using the extended versions of the Prodromal Questionnaire, the translation of these factors to the 16-item screening inventory seems unlikely.

While further replication in larger samples is warranted, our findings go beyond symptom-structure associations by showing the moderating impact of the distress dimension on the anatomical underpinnings of PLE. This posits a crucial distinction for future dimensional model studies as the marked distinction between (positive) subclinical symptoms with varying degrees of subjective impact is stressed. It is currently expected that neuroimaging studies will provide complementary tools for predicting transition to psychosis [92] and long-term clinical outcomes [93]. Our study supports these attempts by isolating the neurobiological 
uniqueness of PLE in the nonclinical part of the psychosis spectrum. We suggest that future investigations might also address the neurobiological characterisation of resilience in genotypes and phenotypes related to psychosis proneness.

Acknowledgements We thank our student research assistants Aliénor Bergmann, Yvonne Schröder, Daniela Hohmann and Franziska Hildesheim for their support.

Author contributions S.G. and I.N. designed the study and obtained funding. U.E., S.S., J.-K.P. and T.M. recruited, assessed, and scanned subjects. T.M. preprocessed MRI data. U.E. performed statistical analysis. I.N. supervised analyses. U.E. wrote the first draft of the manuscript, and all authors contributed to critical revisions of the draft and approved the manuscript.

Funding Open Access funding enabled and organized by Projekt DEAL. Supported by a Research Grant of the University Medical Center Giessen and Marburg (UKGM) (project 11/2017 MR to I.N.; project 05/2018 to Sarah Grezellschak and Igor Nenadić).

Availability of data and materials All original data are on record and accessible to inspection, but are not available publicly based on local and national data protection regulations.

Code availability All software used in the analyses is based on publicly available code.

\section{Compliance with ethical standards}

Conflict of interest The authors declare that they have no conflict of interest.

Open Access This article is licensed under a Creative Commons Attribution 4.0 International License, which permits use, sharing, adaptation, distribution and reproduction in any medium or format, as long as you give appropriate credit to the original author(s) and the source, provide a link to the Creative Commons licence, and indicate if changes were made. The images or other third party material in this article are included in the article's Creative Commons licence, unless indicated otherwise in a credit line to the material. If material is not included in the article's Creative Commons licence and your intended use is not permitted by statutory regulation or exceeds the permitted use, you will need to obtain permission directly from the copyright holder. To view a copy of this licence, visit http://creativecommons.org/licenses/by/4.0/.

\section{References}

1. Schultze-Lutter F, Michel C, Schmidt SJ et al (2015) EPA guidance on the early detection of clinical high risk states of psychoses. Eur Psychiatry 30:405-416. https://doi.org/10.1016/j.eurps y.2015.01.010

2. Schultze-Lutter F, Klosterkötter J, Ruhrmann S (2014) Improving the clinical prediction of psychosis by combining ultra-high risk criteria and cognitive basic symptoms. Schizophr Res 154:100106. https://doi.org/10.1016/j.schres.2014.02.010

3. Fusar-Poli P, Cappucciati M, Rutigliano G et al (2015) At risk or not at risk? A meta-analysis of the prognostic accuracy of psychometric interviews for psychosis prediction. World Psychiatry 14:322-332. https://doi.org/10.1002/wps.20250

4. Kline E, Thompson E, Demro C et al (2015) Longitudinal validation of psychosis risk screening tools. Schizophr Res 165:116122. https://doi.org/10.1016/j.schres.2015.04.026

5. Loewy RL, Bearden CE, Johnson JK et al (2005) The Prodromal Questionnaire (PQ): preliminary validation of a self-report screening measure for prodromal and psychotic syndromes. Schizophr Res 79:117-125. https://doi.org/10.1016/j.schres.2005.03.007

6. Ising HK, Veling W, Loewy RL et al (2012) The validity of the 16-item version of the Prodromal Questionnaire (PQ-16) to screen for ultra high risk of developing psychosis in the general helpseeking population. Schizophr Bull 38:1288-1296. https://doi. org/10.1093/schbul/sbs068

7. Loewy RL, Pearson R, Vinogradov S et al (2011) Psychosis risk screening with the Prodromal Questionnaire - Brief Version (PQB). Schizophr Res 129:42-46. https://doi.org/10.1016/j.schre s.2011.03.029

8. Kline E, Schiffman J (2014) Psychosis risk screening: a systematic review. Schizophr Res 158:11-18. https://doi.org/10.1016/j.schre s.2014.06.036

9. Lorenzo P, Silvia A, Federica P et al (2018) The Italian version of the 16-item prodromal questionnaire (iPQ-16): field-test and psychometric features. Schizophr Res 199:353-360. https://doi. org/10.1016/j.schres.2018.03.023

10. Pelizza L, Azzali S, Paterlini F et al (2019) Screening for psychosis risk among help-seeking adolescents: application of the Italian version of the 16-item prodromal questionnaire (iPQ-16) in child and adolescent neuropsychiatry services. Early Interv Psychiatry 4:752-760. https://doi.org/10.1111/eip.12554

11. Chen F, Wang L, Heeramun-Aubeeluck A et al (2014) Identification and characterization of college students with Attenuated Psychosis Syndrome in China. Psychiatry Res 216:346-350. https ://doi.org/10.1016/j.psychres.2014.01.051

12. McDonald M, Christoforidou E, Van Rijsbergen N et al (2018) Using online screening in the general population to detect participants at clinical high-risk for psychosis. Schizophr Bull 45:600609. https://doi.org/10.1093/schbul/sby069

13. Savill M, D'Ambrosio J, Cannon TD, Loewy RL (2018) Psychosis risk screening in different populations using the Prodromal Questionnaire: a systematic review. Early Interv Psychiatry 12:3-14. https://doi.org/10.1111/eip.12446

14. Linscott RJ, van Os J (2013) An updated and conservative systematic review and meta-analysis of epidemiological evidence on psychotic experiences in children and adults: on the pathway from proneness to persistence to dimensional expression across mental disorders. Psychol Med 43:1133-1149. https://doi.org/10.1017/ S0033291712001626

15. Schultze-Lutter F, Renner F, Paruch J et al (2014) Self-reported psychotic-like experiences are a poor estimate of clinician-rated attenuated and frank delusions and hallucinations. Psychopathology 47:194-201. https://doi.org/10.1159/000355554

16. Falkenberg I, Valmaggia L, Byrnes M et al (2015) Why are helpseeking subjects at ultra-high risk for psychosis help-seeking? Psychiatry Res 228:808-815. https://doi.org/10.1016/j.psych res.2015.05.018

17. McGlashan TH, Miller TJ, Woods SW et al (2010) Structured interview for prodromal syndromes (version 5.0). PRIME Research Clinic, Yale School of Medicine, New Haven, Connecticut

18. Dominguez MDG, Wichers M, Lieb R et al (2011) Evidence that onset of clinical psychosis is an outcome of progressively more persistent subclinical psychotic experiences: an 8-year cohort study. Schizophr Bull 37:84-93. https://doi.org/10.1093/schbul/ $\operatorname{sbp} 022$ 
19. Armando M, Nelson B, Yung AR et al (2010) Psychotic-like experiences and correlation with distress and depressive symptoms in a community sample of adolescents and young adults. Schizophr Res 119:258-265. https://doi.org/10.1016/j.schres.2010.03.001

20. Loewy RL, Johnson JK, Cannon TD (2007) Self-report of attenuated psychotic experiences in a college population. Schizophr Res 93:144-151. https://doi.org/10.1016/j.schres.2007.02.010

21. Hanssen M, Bak M, Bijl R et al (2005) The incidence and outcome of subclinical psychotic experiences in the general population. $\mathrm{Br}$ J Clin Psychol 44:181-191. https://doi.org/10.1348/014466505x 29611

22. Yung AR, Nelson B, Baker K et al (2009) Psychotic-like experiences in a community sample of adolescents: implications for the continuum model of psychosis and prediction of schizophrenia. Aust N Z J Psychiatry 43:118-128. https://doi.org/10.1080/00048 670802607188

23. Brandizzi M, Schultze-Lutter F, Masillo A et al (2014) Selfreported attenuated psychotic-like experiences in help-seeking adolescents and their association with age, functioning and psychopathology. Schizophr Res 160:110-117. https://doi. org/10.1016/j.schres.2014.10.005

24. Fusar-Poli P, Radua J, McGuire P, Borgwardt S (2012) Neuroanatomical maps of psychosis onset: voxel-wise meta-analysis of antipsychotic-naive VBM studies. Schizophr Bull 38:12971307. https://doi.org/10.1093/schbul/sbr 134

25. Smieskova R, Fusar-Poli P, Allen P et al (2010) Neuroimaging predictors of transition to psychosis - a systematic review and meta-analysis. Neurosci Biobehav Rev 34:1207-1222. https:// doi.org/10.1016/j.neubiorev.2010.01.016

26. Nenadić I, Dietzek M, Schönfeld N et al (2015) Brain structure in people at ultra-high risk of psychosis, patients with firstepisode schizophrenia, and healthy controls: a VBM study. Schizophr Res 161:169-176. https://doi.org/10.1016/j.schre s.2014.10.041

27. de Wit S, Wierenga LM, Oranje B et al (2016) Brain development in adolescents at ultra-high risk for psychosis: longitudinal changes related to resilience. NeuroImage Clin 12:542-549. https://doi.org/10.1016/j.nicl.2016.08.013

28. Dietsche B, Kircher T, Falkenberg I (2017) Structural brain changes in schizophrenia at different stages of the illness: a selective review of longitudinal magnetic resonance imaging studies. Aust N Z J Psychiatry 51:500-508. https://doi. org/10.1177/0004867417699473

29. Baumeister D, Sedgwick O, Howes O, Peters E (2017) Auditory verbal hallucinations and continuum models of psychosis: a systematic review of the healthy voice-hearer literature. Clin Psychol Rev 51:125-141. https://doi.org/10.1016/j.cpr.2016.10.010

30. Taylor JH, Calkins ME, Gur RE (2020) Markers of psychosis risk in the general population. Biol Psychiatry 88:337-348. https://doi.org/10.1016/j.biopsych.2020.02.002

31. Nelson MT, Seal ML, Pantelis C, Phillips LJ (2013) Evidence of a dimensional relationship between schizotypy and schizophrenia: a systematic review. Neurosci Biobehav Rev 37:317-327. https://doi.org/10.1016/j.neubiorev.2013.01.004

32. Esterberg ML, Compton MT (2009) The psychosis continuum and categorical versus dimensional diagnostic approaches. Curr Psychiatry Rep 11:179-184. https://doi.org/10.1007/s 1192 0-009-0028-7

33. Allardyce J, Suppes T, van Os J (2007) Dimensions and the psychosis phenotype. Int J Methods Psychiatr Res 16(Suppl 1):S34-40. https://doi.org/10.1002/mpr.214

34. Nenadić I, Lorenz C, Langbein K et al (2015) Brain structural correlates of schizotypy and psychosis proneness in a non-clinical healthy volunteer sample. Schizophr Res 168:37-43. https ://doi.org/10.1016/j.schres.2015.06.017
35. Waltmann M, O’Daly O, Egerton A et al (2018) Multi-echo fMRI, resting-state connectivity, and high psychometric schizotypy. NeuroImage Clin 21:1-9. https://doi.org/10.1016/j. nicl.2018.11.013

36. Evermann U, Gaser C, Besteher B et al (2020) Cortical gyrification, psychotic-like experiences, and cognitive performance in nonclinical subjects. Schizophr Bull 46:1524-1534. https://doi. org/10.1093/schbul/sbaa068

37. Satterthwaite TD, Wolf DH, Calkins ME et al (2016) Structural brain abnormalities in youth with psychosis spectrum symptoms. JAMA Psychiatry 73:515-524. https://doi.org/10.1001/jamap sychiatry.2015.3463

38. Meller T, Schmitt S, Ettinger U, et al (2020) Brain structural correlates of schizotypal signs and subclinical schizophrenia nuclear symptoms in healthy individuals. Psychol Med 1-10. https://doi. org/10.1017/S0033291720002044

39. Pfarr J-K, Nenadić I (2020) A multimodal imaging study of brain structural correlates of schizotypy dimensions using the MSS. Psychiatry Res Neuroimaging 302:111104. https://doi. org/10.1016/j.pscychresns.2020.111104

40. Lenzenweger MF (2006) Schizotypy: an organizing framework for schizophrenia research. Curr Dir Psychol Sci 15:162-166. https:// doi.org/10.1111/j.1467-8721.2006.00428.x

41. Gottesman II, Gould TD (2003) The endophenotype concept in psychiatry: etymology and strategic intentions. Am J Psychiatry 160:636-645. https://doi.org/10.1176/appi.ajp.160.4.636

42. Kline E, Wilson C, Ereshefsky S et al (2012) Schizotypy, psychotic-like experiences and distress: an interaction model. Psychiatry Res 200:647-651. https://doi.org/10.1016/j.psych res.2012.07.047

43. Barrantes-Vidal N, Grant P, Kwapil TR (2015) The role of schizotypy in the study of the etiology of schizophrenia spectrum disorders. Schizophr Bull 41:S408-S416. https://doi.org/10.1093/ schbul/sbu191

44. Barrantes-Vidal N, Lewandowski KE, Kwapil TR (2010) Psychopathology, social adjustment and personality correlates of schizotypy clusters in a large nonclinical sample. Schizophr Res 122:219-225. https://doi.org/10.1016/j.schres.2010.01.006

45. Grant P, Gabriel F, Kuepper Y et al (2014) Psychosis-proneness correlates with expression levels of dopaminergic genes. Eur Psychiatry 29:304-306. https://doi.org/10.1016/j.eurpsy.2013.12.002

46. Grant P, Kuepper Y, Mueller EA et al (2013) Dopaminergic foundations of schizotypy as measured by the German version of the Oxford-Liverpool Inventory of Feelings and Experiences (O-LIFE) - a suitable endophenotype of schizophrenia. Front Hum Neurosci 7:1-11. https://doi.org/10.3389/fnhum.2013.00001

47. Howes OD, Kapur S (2009) The dopamine hypothesis of Schizophrenia: version III-the final common pathway. Schizophr Bull 35:549-562. https://doi.org/10.1093/schbul/sbp006

48. Dandash O, Pantelis C, Fornito A (2017) Dopamine, frontostriato-thalamic circuits and risk for psychosis. Schizophr Res 180:48-57. https://doi.org/10.1016/j.schres.2016.08.020

49. Meller T, Ettinger U, Grant P, Nenadić I (2020) The association of striatal volume and positive schizotypy in healthy subjects: intelligence as a moderating factor. Psychol Med 50:2355-2363. https://doi.org/10.1017/S0033291719002459

50. World Medical Association (2013) World Medical Association Declaration of Helsinki: ethical principles for medical research involving human subjects. JAMA 310:2191-2194. https://doi. org/10.1001/jama.2013.281053

51. Wittchen H-U, Wunderlich U, Gruschwitz S, Zaudig M (1997) SKID-I. Strukturiertes Klinisches Interview für DSM-IV. Hogrefe, Göttingen

52. Leiner DJ (2019) SoSci Survey (Version 3.1.06) [Computer software]. https://www.soscisurvey.de 
53. Oldfield RC (1971) The assessment and analysis of handedness: the Edinburgh inventory. Neuropsychologia 9:97-113. https://doi. org/10.1016/0028-3932(71)90067-4

54. Lehrl S (2005) Mehrfachwahl-Wortschatz-Intelligenztest MWT-B. Spitta Verlag, Balingen

55. Chen F, Wang L, Wang J et al (2016) Applicability of the Chinese version of the 16-item Prodromal Questionnaire (CPQ-16) for identifying attenuated psychosis syndrome in a college population. Early Interv Psychiatry 10:308-315. https://doi.org/10.1111/ eip. 12173

56. Kotzalidis GD, Solfanelli A, Piacentino D et al (2017) The Italian version of the 92-item Prodromal Questionnaire: concurrent validity with the SIPS and factor analysis in a sample of 258 outpatients aged 11-36 years. Schizophr Res 189:50-56. https://doi. org/10.1016/j.schres.2017.02.008

57. Hayes AF (2018) Introduction to mediation, moderation, and conditional process analysis: a regression-based approach, 2nd edn. Guilford Press, New York, NY

58. Benjamini Y, Hochberg Y (1995) Controlling the false discovery rate: a practical and powerful approach to multiple testing. J R Stat Soc Ser B 57:289-300

59. R Core Team (2020) R: a language and environment for statistical computing. R Foundation for Statistical Computing, Vienna. https ://www.r-project.org/

60. Schultze-Lutter F, Addington J, Ruhrmann S, Klosterkötter J (2007) Schizophrenia proneness instrument, Adult Version (SPIA). Giovanni Fioriti Editore, Roma

61. Simon AE, Borgwardt S, Riecher-Rössler A et al (2013) Moving beyond transition outcomes: meta-analysis of remission rates in individuals at high clinical risk for psychosis. Psychiatry Res 209:266-272. https://doi.org/10.1016/j.psychres.2013.03.004

62. Fusar-Poli P, Cappucciati M, Borgwardt S et al (2016) Heterogeneity of psychosis risk within individuals at clinical high risk: a meta-analytical stratification. JAMA Psychiatry 73:113-120. https ://doi.org/10.1001/jamapsychiatry.2015.2324

63. Honea R, Crow TJ, Passingham D, Mackay CE (2005) Regional deficits in brain volume in schizophrenia: a meta-analysis of voxel-based morphometry studies. Am J Psychiatry 162:22332245. https://doi.org/10.1176/appi.ajp.162.12.2233

64. van Erp TGM, Walton E, Hibar DP et al (2018) Cortical brain abnormalities in 4474 individuals with schizophrenia and 5098 control subjects via the enhancing neuro imaging genetics through meta analysis (ENIGMA) consortium. Biol Psychiatry 84:644654. https://doi.org/10.1016/j.biopsych.2018.04.023

65. Modinos G, Costafreda SG, Van Tol MJ et al (2013) Neuroanatomy of auditory verbal hallucinations in schizophrenia: a quantitative meta-analysis of voxel-based morphometry studies. Cortex 49:1046-1055. https://doi.org/10.1016/j.cortex.2012.01.009

66. Nenadić I, Maitra R, Langbein K et al (2015) Brain structure in schizophrenia vs. psychotic bipolar I disorder: a VBM study. Schizophr Res 165:212-219. https://doi.org/10.1016/j.schre s.2015.04.007

67. Nenadić I, Sauer H, Gaser C (2010) Distinct pattern of brain structural deficits in subsyndromes of schizophrenia delineated by psychopathology. Neuroimage 49:1153-1160. https://doi. org/10.1016/j.neuroimage.2009.10.014

68. Fusar-Poli P, Borgwardt S, Crescini A et al (2011) Neuroanatomy of vulnerability to psychosis: a voxel-based meta-analysis. Neurosci Biobehav Rev 35:1175-1185. https://doi.org/10.1016/j.neubi orev.2010.12.005

69. Ohtani T, Levitt JJ, Nestor PG et al (2014) Prefrontal cortex volume deficit in schizophrenia: a new look using 3T MRI with manual parcellation. Schizophr Res 152:184-190. https://doi. org/10.1016/j.schres.2013.10.026

70. Chang M, Womer FY, Bai C et al (2016) Voxel-based morphometry in individuals at genetic high risk for schizophrenia and patients with schizophrenia during their first episode of psychosis. PLoS ONE 11:1-12. https://doi.org/10.1371/journal.pone.01637 49

71. Legge SE, Jones HJ, Kendall KM et al (2019) Association of genetic liability to psychotic experiences with neuropsychotic disorders and traits. JAMA Psychiatry 76:1256-1265. https://doi. org/10.1001/jamapsychiatry.2019.2508

72. Andreasen NC, Nopoulos P, Magnotta V et al (2011) Progressive brain change in schizophrenia: a prospective longitudinal study of first-episode schizophrenia. Biol Psychiatry 70:672-679. https ://doi.org/10.1016/j.biopsych.2011.05.017

73. Ziermans TB, Schothorst PF, Schnack HG et al (2012) Progressive structural brain changes during development of psychosis. Schizophr Bull 38:519-530. https://doi.org/10.1093/schbul/sbq11 3

74. Pantelis C, Yücel M, Wood SJ et al (2005) Structural brain imaging evidence for multiple pathological processes at different stages of brain development in schizophrenia. Schizophr Bull 31:672696. https://doi.org/10.1093/schbul/sbi034

75. Bartholomeusz CF, Cropley VL, Wannan C et al (2017) Structural neuroimaging across early-stage psychosis: aberrations in neurobiological trajectories and implications for the staging model. Aust N Z J Psychiatry 51:455-476. https://doi. org/10.1177/0004867416670522

76. Klauser P, Zhou J, Lim JKW et al (2015) Lack of evidence for regional brain volume or cortical thickness abnormalities in youths at clinical high risk for psychosis: findings from the longitudinal youth at risk study. Schizophr Bull 41:1285-1293. https://doi.org/10.1093/schbul/sbv012

77. Siever LJ, Davis KL (2004) The pathophysiology of schizophrenia disorders: perspectives from the spectrum. Am J Psychiatry 161:398-413. https://doi.org/10.1176/appi.ajp.161.3.398

78. Cannon TD, Chung Y, He G et al (2015) Progressive reduction in cortical thickness as psychosis develops: a multisite longitudinal neuroimaging study of youth at elevated clinical risk. Biol Psychiatry 77:147-157. https://doi.org/10.1016/j.biops ych.2014.05.023

79. Modinos G, Mechelli A, Ormel J et al (2010) Schizotypy and brain structure: a voxel-based morphometry study. Psychol Med 40:1423-1431. https://doi.org/10.1017/S0033291709991875

80. Modinos G, Egerton A, McLaughlin A et al (2018) Neuroanatomical changes in people with high schizotypy: relationship to glutamate levels. Psychol Med 48:1880-1889. https://doi. org/10.1017/S0033291717003403

81. Fusar-Poli P, Smieskova R, Serafini G et al (2014) Neuroanatomical markers of genetic liability to psychosis and first episode psychosis: a voxelwise meta-analytical comparison. World J Biol Psychiatry 15:219-228. https://doi.org/10.3109/15622 975.2011.630408

82. Borgwardt SJ, McGuire PK, Aston J et al (2007) Structural brain abnormalities in individuals with an at-risk mental state who later develop psychosis. Br J Psychiatry Suppl 51:s69-75. https ://doi.org/10.1192/bjp.191.51.s69

83. Premkumar P, Kumari V, Corr PJJ et al (2008) Neuropsychological function - brain structure relationships and stage of illness : an investigation into chronic and first-episode schizophrenia. Psychiatry Res 162:195-204. https://doi.org/10.1016/j.pscyc hresns.2007.08.005

84. Barahmand U, Heydari Sheikh Ahmad R (2016) Psychoticlike experiences and psychological distress: the role of resilience. J Am Psychiatr Nurses Assoc 22:312-319. https://doi. org/10.1177/1078390316653802

85. Ghuman AS, Brunet NM, Li Y et al (2014) Dynamic encoding of face information in the human fusiform gyrus. Nat Commun 5:5672. https://doi.org/10.1038/ncomms6672 
86. Haxby JV, Hoffman EA, Gobbini MI (2000) The distributed human neural system for face perception. Trends Cogn Sci 4:223-233. https://doi.org/10.1016/s1364-6613(00)01482-0

87. Lee CU, Shenton ME, Salisbury DF et al (2002) Fusiform gyrus volume reduction in first-episode schizophrenia: a magnetic resonance imaging study. Arch Gen Psychiatry 59:775-781. https ://doi.org/10.1001/archpsyc.59.9.775

88. Whittaker JF, Deakin JFW, Tomenson B (2001) Face processing in schrizophrenia: defining the deficit. Psychol Med 31:499507. https://doi.org/10.1017/s0033291701003701

89. Guo X, Li J, Wei Q et al (2013) Duration of untreated psychosis is associated with temporal and occipitotemporal gray matter volume decrease in treatment naïve schizophrenia. PLoS One 8:e83679. https://doi.org/10.1371/journal.pone.0083679

90. Onitsuka T, Shenton ME, Kasai K et al (2003) Fusiform gyrus volume reduction and facial recognition in chronic schizophrenia. Arch Gen Psychiatry 60:349-355. https://doi.org/10.1001/ archpsyc.60.4.349
91. Unterrassner L, Wyss TA, Wotruba D et al (2017) Psychoticlike experiences at the healthy end of the psychosis continuum. Front Psychol 8:1-13. https://doi.org/10.3389/fpsyg.2017.00775

92. Andreou C, Borgwardt S (2020) Structural and functional imaging markers for susceptibility to psychosis. Mol Psychiatry 25:27732785. https://doi.org/10.1038/s41380-020-0679-7

93. de Wit S, Ziermans TB, Nieuwenhuis M et al (2017) Individual prediction of long-term outcome in adolescents at ultra-high risk for psychosis: applying machine learning techniques to brain imaging data. Hum Brain Mapp 38:704-714. https://doi. org/10.1002/hbm. 23410

94. Wickham H (2016) ggplot2: elegant graphics for data analysis. Springer-Verlag, New York, NY

95. Kikinis R, Pieper SD, Vosburgh KG (2014) 3D Slicer: a platform for subject-specific image analysis, visualization, and clinical support. In: Jolesz FA (ed) BT-intraoperative imaging and imageguided therapy. Springer New York, New York, NY, pp 277-289 